Антигипертензивная эффективность олмесартана

медоксомила и кандесартана цилексетила в отношении снижения артериального давления в течение суток и достижения целевых уровней давления по данным суточного амбулаторного мониторирования*

\author{
Х.Р. Бруннер ${ }^{1}$, К. Аракава ${ }^{2}$
}

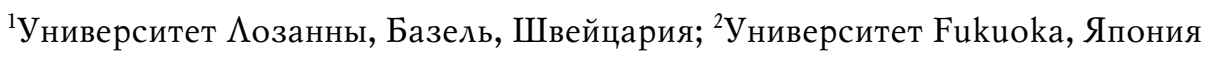

\title{
Antihypertensive efficacy of olmesartan medoxomil and candesartan cilexetil in achieving 24-hour blood pressure reductions and ambulatory blood pressure goals*
}

\author{
Hans R. Brunner ${ }^{1}$, Kikuo Arakawa ${ }^{2}$ \\ ${ }^{1}$ Lausanne University, Medizinische Poliklinik, Universitaetsspital, Basel, Switzerland; ${ }^{2}$ Fukuoka University, \\ Fukuoka, Japan
}

Введение. У пациентов с гипертензией (АГ) эффективный контроль артериального давления (АД) на протяжении всех сут. играет важную роль в профилактике утреннего подъема (УП) АД и, соответственно, в снижении риска сердечно-сосудистых осложнений (ССО). Целью исследования было сравнение суточной антигипертензивной эффективности олмесартана медоксомила (ОМ) и кандесартана цилексетила (КЦ) с фокусом на эффективность этих препаратов в отношении контроля АД в ранние утренние часы.

Методы. Настоящее исследование - дополнительный анализ данных ранее выполненного, рандомизированного, двойного слепого исследования, в котором 635 пациентов с мягкой и умеренной АГ рандомизировались в отношении 8-недельной терапии ОМ (20 мг/сут.) либо КЦ (8 мг/сут.). Через 1, 2 и 8 нед. лечения в обеих группах оценивали снижение исходных уровней АД в последние 2 и 4 ч суточного амбулаторного мониторирования АД (СМАД). Оценивалась доля пациентов с достижением целевых уровней АД по критериям ESH/ESC (<120/80 мм рт.ст.) и по критериям JSH (<135/80 мм рт.ст.). В обеих группах проводилась оценка достижения целевых уровней АД в течение всех суток, в дневные часы, а также в последние 4 и 2 ч СМ.

Результаты. Через 8 нед. терапии доля пациентов с достижением целевых уровней суточного (24) и дневного (д) амбулаторного АД была достоверно выше среди принимавших ОМ, чем среди получавших КЦ. Межгрупповые различия достигали статистической значимости при использовании как критериев ESH/ ESC: $25,6 \%$ vs $14,9 \%$ для АД 24 ( $p<0,001) ; 18,3 \%$ vs $9,6 \%$ для целевого АДд $(p=0,002)$ ), так и критериев JSH: $37,5 \%$ vs $26,6 \%$ для суточного целевого АД ( $p=0,003) ; 26,6 \%$ vs $16,4 \%$ для целевого АДд $(p=0,002)$. Частота достижения целевых уровней давления в последние 4 ч СМ была значимо выше в группе ОМ $-33,3 \%$ и 39,1 \% по критериям ESH/ESC и JSH, соответственно, чем в группе КЦ - 22,9\% $(p<0,001)$ и $31,6 \%$ ( $p=0,047)$, соответственно. Аналогично, различия по частоте достижения целевых уровней АД в последние 2 ч СМАД были достоверными (по критериям JSH) либо близкими к статистически достоверным (по критериям ESH/ESC), при сравнении группы OM - 26,9\% и 19,9\%, соответственно, с группой КЦ $-19,6 \%(p=0,028)$ и $14,3 \%(p=0,061)$, соответственно.

Заключение. Терапия ОМ ассоциировалась с большей частотой достижения целевых уровней давления по данным CMAД, как по критериям ESH/ESC, так и по критериям JSH. Более эффективный контроль АД при лечении ОМ также подтверждался большей частотой достижения целевых уровней АД по критериям ESH/ESC и JSH в ранние утренние часы. Это не только свидетельствует о более эффективном суточном контроле давления при приеме ОМ, но и позволяет предположить, что препарат обеспечивает большее снижение риска ССО, ассоциирующегося с УП АД.

Background. For patients with hypertension, effective 24-hour blood pressure (BP) control is vital to ensure protection against the early morning surge in BP and the associated increased risk of cardiovascular events. The aim of this analysis was to assess the 24-hour antihypertensive efficacy of olmesartan medoxomil (20 mg once daily) compared with candesartan cilexetil ( $8 \mathrm{mg}$ once daily), with particular emphasis on BP control during the early morning period.

Methods. This is an additional analysis of a previously reported randomised, double-blind study in which

[*Адаптированный перевод из журнала Clin Drug Invest 2006; 26(4): 185-193]. 
635 patients with mainly mild to moderate hypertension were randomised to 8 weeks of treatment with either olmesartan medoxomil $20 \mathrm{mg} /$ day or candesartan cilexetil $8 \mathrm{mg} /$ day. Changes from baseline during the last 4 and 2 hours of ambulatory BP measurement (ABPM) after 1, 2 and 8 weeks of treatment were compared between the two groups. In addition, the proportions of patients who achieved various ABPM goals, including those suggested by the European Society of Hypertension/European Society of Cardiology (ESH/ESC) [<125/80 mm Hg] and the Japanese Society of Hypertension (JSH) $[<135 / 80 \mathrm{~mm} \mathrm{Hg}]$, over 24 hours, during the daytime and at the last 4 and 2 hours of ABPM measurement were also compared.

Results. After 8 weeks, significantly greater proportions of patients treated with olmesartan medoxomil $20 \mathrm{mg}$ achieved 24-hour and daytime ABPM goals recommended by the guidelines of the ESH/ESC (25,6\% and 18,3\%, respectively) and JSH (37,5\% and 26,6\%, respectively) compared with candesartan cilexetil $8 \mathrm{mg}$ (24-hour ESH/ ESC goal 14,9\%, $p<0,001$; 24-hour JSH goal 26,6\%, $p=0,003$; daytime ESH/ESC goal 9,6\%, $p=0,002$; daytime JSH goal $16,4 \%, p=0,002$ ). During the last 4 hours of 24-hour ABPM, the proportions of patients who achieved the ESH/ESC and JSH ABPM goals were significantly greater with olmesartan medoxomil $(33,3 \%$ and $39,1 \%$, respectively) than with candesartan cilexetil $(22,9 \%, p<0,001$ and $31,6 \%, p=0,047$, respectively). Similarly, during the last 2 hours of 24-hour ABPM, the proportions of patients who achieved these BP goals were either significantly greater (JSH) or approached statistical significance (ESH/ESC) with olmesartan medoxomil (26,9 \% and $19,9 \%$, respectively), compared with candesartan cilexetil $(19,6 \%, p=0,028$ and $14,3 \%, p=0,061$, respectively).

Conclusion. Compared with candesartan cilexetil $8 \mathrm{mg}$, greater proportions of olmesartan medoxomil-treated patients $(20 \mathrm{mg})$ achieved ESH/ESC and JSH ABPM goals over 24 hours. The superior BP control of olmesartan medoxomil was also reflected in the larger proportions of olmesartan medoxomil-treated patients who achieved the ESH/ESC and JSH ABPM goals during the early morning surge period. This not only demonstrates that olmesartan medoxomil $20 \mathrm{mg}$ provides superior 24-hour BP reduction, but also suggests that olmesartan medoxomil may provide greater protection against the increased risk of cardiovascular events associated with the early morning BP surge period.

Известно, что гипертензия (АГ) является ведущим фактором риска (ФР) заболеваемости и преждевременной смертности [1], в первую очередь, от сердечно-сосудистых заболеваний (CC3) [2,3]. Изменения артериального давления (АД) в течение суток сопровождаются соответствующими изменениями риска сердечно-сосудистых осложнений (ССО) [4-6]. В частности, наблюдаемый в утренние часы (08:00-12:00) подъем (УП) АД ассоциируется с увеличением частоты сердечно-сосудистых событий (ССС) [5,7]. В крупных клинических исследованиях был убедительно продемонстрирован благоприятный эффект контроля АД в отношении снижения сердечно-сосудистого риска (ССР) [8-10]. В современных международных и национальных клинических руководствах, таких как рекомендации European Society of Hypertension, European Society of Cardiology (ESH/ESC) [11] и Japanese Society of Hypertension (JSH) [12] подчеркивается важность снижения АД, а также приводятся целевые уровни АД [11-13]. У пациентов с АГ важно обеспечить как адекватный контроль АД на протяжении всех суток, так и предупреждение УП АД [7].

В большинстве клинических исследований антигипертензивной терапии (АГТ) используется традиционный манжеточный метод измерения АД. Для оценки АД также может применяться амбулаторное мониторирование (АМ) АД, при котором производятся регулярные измерения АД в течение определенного периода времени. По сравнению с традиционным методом, АМ АД характеризуется целым рядом преимуществ, в т.ч. независимостью показателей от эффекта “белого халата" [14] и более точной оценкой ССР [15,16] и риска поражения органов-мишеней (ПОМ) [17].

Олмесартан медоксомил (ОМ) является новейшим представителем такого класса антигипертензивных препаратов (АГП), как блокаторы рецепторов к ангиотензину II (АТ ІІ) (БРА). Как было показано ранее, ОМ обладает высокой антигипертензивной эффективностью. Он является единственным БРА, который, по результатам прямых сравнительных исследований официально рекомендуе- мых начальных доз, более эффективно снижает среднее диастолическое АД (ДАДср), чем 4 других БРА (кандесартан цилексетил, лозартан, валсартан и ирбесартан) [18, 19]. Выполненные в Японии рандомизированные клинические испытания (РКИ) показали, что ОМ достоверно более эффективен в отношении снижения АД, чем 3 упомянутых выше БРА, а также БРА телмисартан [20]. В одном исследовании, непосредственно сравнивавшем терапию ОМ (20 мг/сут. один раз) и лечение кандесартаном цилексетилом (КЦ) (8 мг/сут. один раз) у пациентов с мягкой и умеренной эссенциальной АГ, оценка антигипертензивной эффективности выполнялась по данным суточного АМ АД (СМАД). Было показано, что прием ОМ ассоциировался с более выраженным средним снижением суточных уровней ДАД и систолического АД (САД) [18]. При этом влияние терапии на предупреждение УП АД не оценивалось. В настоящей статье представлены результаты дополнительного анализа результатов этого исследования. В частности, выполнена оценка влияния АГТ на предупреждение УП АД, с определением частоты достижения целевого АД в течение суток и в утренние часы, согласно критериям ESH/ESC (2003) и JSH (2004) [11,12].

\section{Методы}

Поскольку дизайн исследования был подробно описан ранее [18], в этой статье приводится его краткая характеристика.

Дизайн исследования и характеристика участников исследования. Многоцентровое, рандомизированное, двойное слепое исследование в параллельных группах было выполнено на базе 44 клинических центров Германии, Польши и Чехии. Исследование проводилось в соответствии с принципами качественной клинической практики (Good Clinical Practice) и Хельсинкской Декларации. Протокол исследования был одобрен этическими комитетами всех участвующих центров. Перед включением в исследование 
у всех пациентов было получено письменное информированное согласие.

Во время скрининга у потенциальных участников мужчин и женщин > 18 лет, проводилось физикальное обследование и сбор анамнеза. Основным критерием включения в исследование были измеренные в положении сидя средние уровни остаточного ДАД 100-120 мм рт.ст. и остаточного САД $>150$ мм рт.ст. Для пациентов, отвечавших критериям включения и не принимавших АГП, 2-недельная фаза включения представляла собой простой слепой прием плацебо. У больных, отвечавших критериям включения и получавших АГТ, 2-недельной фазе включения предшествовала постепенная (1-2 нед.) отмена ранее назначенной терапии. По окончанию простой слепой фазы включения, начиналась фаза двойной слепой терапии, при условии соответствия пациентов критериям включения: ДАД 100120 мм рт.ст., САД $>150$ мм рт.ст, средний уровень ДАД 24 $\geq 84$ мм рт.ст., при $\geq 30 \%$ ДАДд $>90$ мм рт.ст.

Больные рандомизировались в отношении двойной слепой терапии ОМ (20 мг/сут. 1 раз) либо КЦ (8 мг/сут. 1 раз). Препараты принимались непосредственно перед завтраком, за исключением дней, когда выполнялись визиты в клинику, и прием препаратов имел место по завершении программы обследования. Во время исследования запрещался прием иных АГП, а также ограничивалось использование других лекарственных средств [18].

Оценка уровней АД с помощью СМАД выполнялась в начале фазы двойной слепой терапии, а также через 1, 2 и 8 нед. лечения. Мониторирование начиналось в 06:00, с измерениями АД через каждые 15 мин, вплоть до 22:00. В ночное время (22:00-06:00) измерения давления выполнялись через каждые 30 мин. В анализ включались лишь показатели СМАД, содержавшие $\geq 64$ суточных и $\geq 52$ дневных измерений. Рутинное измерение АД выполнялось перед завтраком, до приема препаратов.

Анализ данных включал определение доли пациентов с достижением целевых уровней АД 24 и АДд по результатам СМАД, в соответствии с критериями ESH/ESC и JSH $[11,12]$. Также оценивалась доля больных с достижением целевых значений АД в последние 4 и 2 ч СМАД, по критериям ESH/ESC и JSH. Определялась доля лиц, которые в последние 2 ч СМАД достигали целевых уровней АД, рекомендуемых JSH для пожилых пациентов (<140/90 мм рт.

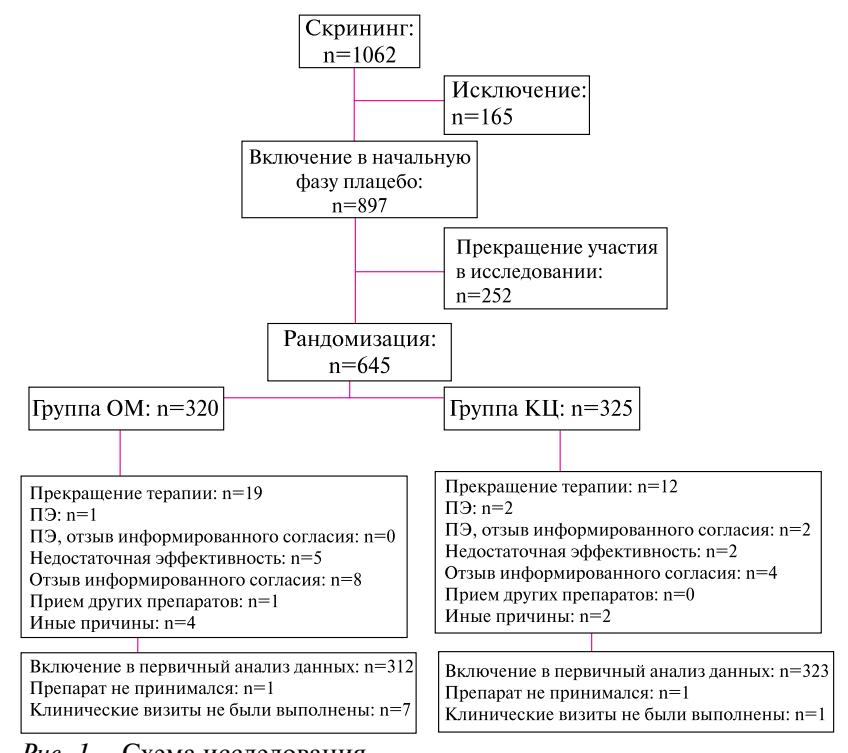

ст.), для больных среднего имолодого возраста (<130/85 мм рт. ст.) и для больных с патологией почеклибо СД (<130/80 мм рт. ст.).

Следует отметить, что в данном анализе использовались суточные целевые уровни АД при СМАД, рекомендуемые ESH/ESC и JSH. Ни одной из этих организаций не были предложены целевые уровни АД, специфичные для последних 4 либо 2 ч СМАД [11,12].

Безопасность и переносимость терапии. На протяжении всего исследования выполнялась регистрация побочных эффектов (ПЭ), сообщаемых пациентами либо наблюдаемых врачами-исследователями. На этапе скрининга, во время фазы терапии и по окончанию лечения выполнялись рутинные лабораторные исследования и электрокардиография (ЭКГ).

Статистический анализ. Частота достижения целевых уровней давления по данным СМАД сравнивалась с помощью критерия $\chi^{2}$.

\section{Результаты}

Участники исследования. В целом, 645 пациентов были рандомизированы в отношении двойной слепой терапии ОМ либо КЦ (рисунок 1). В анализ в группе рандомизации (intention-to-treat) вошли данные 635 больных: 312 из группы ОМ и 323 из группы КЦ. Полностью выполнили протокол исследования 614 участников: 301 из группы ОМ и 313 из группы КЦ. Обе группы терапии были сопоставимы по демографическим и клиническим характеристикам [18]. Средняя длительность АГ была несколько выше в группе ОМ, чем в группе КЦ. Исходные показатели СМАД были сопоставимыми в обеих группах рандомизации (таблица 1).

Эффективность. Ранее было показано, что терапия ОМ по сравнению с приемом КЦ ассоциировалась с более выраженным средним снижением уровней ДАД и САД, по данным выполненного на 1, 2 и 8 нед. СМАД [18]. К концу 8 нед. доля пациентов с достижением целевых АД 24 при СМАД была достоверно выше в группе ОМ, чем в группе КЦ, как по критериям $\operatorname{ESH} / \operatorname{ESC}(p=0,003)$, так и по критериям JSH $(p<0,001)$ (рисунок 2). Доля больных с достижением целевых уровней АДд СМАД (06:00-22:00) по критериям ESH/ESC и JSH была достоверно выше при терапии ОМ, чем при лечении КЦ ( $p=0,002$ для обоих критериев) (рисунок 2).

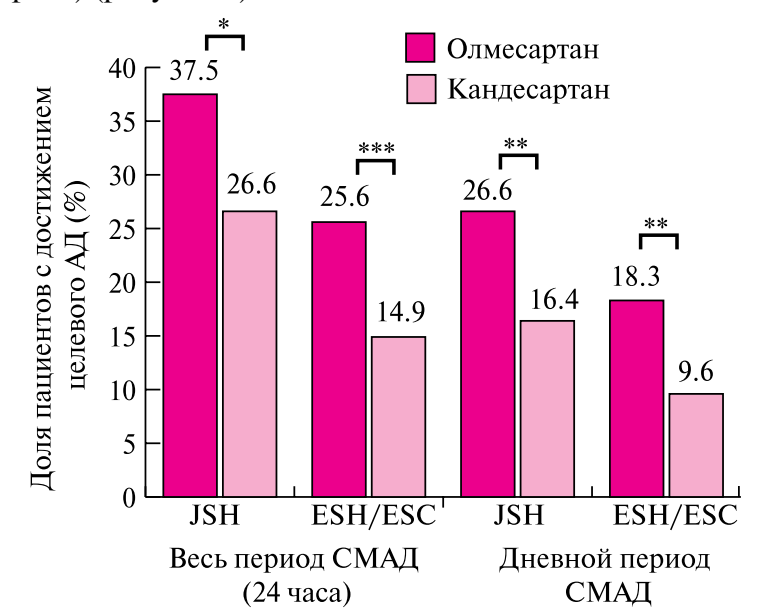

Примечание: * $p=0,003 ;{ }^{* *} p=0,002 ;{ }^{* * *} p<0,001$.

Puc. 2 Доля пациентов с достижением целевых АД 24 и АДд при СМАД на 8 нед. терапии по критериям JSH (<135/80 мм рт. ст.; [12]) и ЕSH/ESC (<125/80 мм рт.ст.; [11]). 
Таблица 1

Исходные демографические и клинические характеристики пациентов в группе рандомизации

\begin{tabular}{llll}
\hline Показатель & Группа ОМ (n=312) & Группа КЦ $(\mathrm{n}=323)$ & \multicolumn{1}{c}{$\begin{array}{l}\text { Все участники исследования } \\
(\mathrm{n}=635)\end{array}$} \\
\hline Средний возраст (лет) & $51,5 \pm 10,5$ & $51,8 \pm 9,8$ & $51,7 \pm 10,1$ \\
Возраст (лет) & $19-86$ & $20-76$ & $19-86$ \\
Мужчины (\%) & 56,4 & 56,7 & 56,5 \\
Длительность АГ (лет) & 7,5 & 6,6 & 7,1 \\
& & Параметры СМАД & $92,3 \pm 4,9$ \\
ДАД 24 (мм рт. ст.) & $92,3 \pm 4,9$ & $92,2 \pm 4,9$ & $95,1 \pm 5,2$ \\
ДАДд & $95,1 \pm 5,2$ & $95,0 \pm 5,2$ & $81,5 \pm 7,4$ \\
(мм рт.ст.) & & & $146,0 \pm 10,9$ \\
ДАДн & $81,7 \pm 7,5$ & $81,4 \pm 7,3$ & $149,2 \pm 11,1$ \\
(мм рт.ст.) & $145,7 \pm 11$ & & $133,9 \pm 13,3$ \\
САД 24 (мм рт.ст.) & $148,9 \pm 11,2$ & $146,3 \pm 10,7$ & $104,6 \pm 3,7$ \\
САДд (мм рт.ст.) & $133,6 \pm 14$ & $149,4 \pm 11,1$ & $162,5 \pm 9,3$ \\
САДн (мм рт.ст.) & \multicolumn{1}{c}{ Параметры рутинного измерения остаточного АД ${ }^{\text {b }}$} \\
ДАД в положении сидя & $104,9 \pm 3,8$ & $104,4 \pm 3,6$ & $162,4 \pm 9,2$ \\
САД в положении сидя & $162,5 \pm 9,5$ & $16,2 \pm 12,6$ & \\
\hline
\end{tabular}

а) Данные представлены в виде “среднее значение \pm стандартное отклонение”, если не указано иное. b) Измерение АД выполнялось через $24 \pm 2$ ч от последнего приема препарата, с помощью стандартного ртутного сфигмоманометра.

Предупреждение УП АД. Для более полной оценки эффекта терапии на контроль АД в течение суток было выполнено сравнение влияния приема ОМ и КЦ на величину ДАД и САД в последние 4 и 2 ч СМАД, по окончанию 1 , 2 и 8 нед. лечения. Было показано, что среднее снижение исходных ДАД и САД в последние 4 и 2 ч СМАД было более выраженным в группе ОМ, чем в группе КЦ. Эти различия достигали статистической значимости по окончании 2 недель терапии (рисунок 3). Среди участников, принимавших ОМ, также была выше доля больных со стойким снижением АД. По окончанию 8 нед. лечения доля лиц с достижением целевых значений АД в последние 4 ч СМАД была значимо выше в группе ОМ, чем в группе КЦ, как по критериям $\mathrm{ESH} / \mathrm{ESC}(p<0,001)$, так и по критериям JSH $(p=0,047)$ (рисунок 4). Независимо от критериев целевых уровней АД, по окончанию 8 нед. лечения доля больных, у которых удалось добиться достижения целевого АД в последние 2 ч СМАД, была выше в группе ОМ, по сравнению с группой КЦ (таблица 2). Межгрупповые различия либо были статистически значимыми (при использовании критериев ESH/ $\mathrm{ESC} ; p=0,028)$, либо приближались к статистически значимым (при использовании критериев JSH; $p=0,061$ ).

Безопасность и переносимость терапии. ПЭ были зарегистрированы у 30 принимавших ОМ пациентов и у 40 получавших КЦ. Число ПЭ, связанных с терапией, составило 13
$(4,1 \%)$ и $21(6,5 \%)$, соответственно [18]. На протяжении всего периода наблюдения отсутствовали тяжелые ПЭ, связанные с лечением, а также клинически значимые изменения лабораторных показателей либо ЭКГ параметров.

\section{Обсуждение}

В настоящей статье представлены результаты дополнительного анализа данных ранее выполненного клинического исследования. В нем сравнивалась антигипертензивная эффективность ОМ (20 мг/сут) и КЦ (8 мг/сут) у пациентов с мягкой и умеренной гипертензией [18]. Эти дозы были выбраны, как наиболее подходящие для сравнительного анализа. В Европе рекомендуемые дозы ОМ составляют 10-40 мг/сут., при поддерживающей дозе 20 мг/сут. Во время выполнения исследования, рекомендуемые дозы КЦ составляли 4-16 мг/сут., при поддерживающей дозе 8 мг/сут. В публикации, впервые представившей результаты исследования, было показано, что терапия ОМ (20 мг/сут. однократно) ассоциировалась с более выраженным средним снижением АД по данным СМАД, чем прием КЦ (8 мг/сут.) [18]. Согласно результатам дополнительного анализа, ОМ обеспечивал более эффективное снижение уровней АД 24 , что подтверждалось большей долей пациентов с достижением целевых значений АД по критериям ESH/ESC и JSH. Было продемонстрировано, что более выраженное снижение

Таблица 2

Доля пациентов с достижением целевых уровней АД в последние 2 ч СМАД, к концу 8 нед. терапии ОМ либо КЦ

\begin{tabular}{|c|c|c|c|}
\hline $\begin{array}{l}\text { Целевые уровни АД } \\
\text { в последние } 2 \text { ч СМАД }\end{array}$ & $\begin{array}{l}\text { Группа ОМ } \\
\text { (20 мг/сут.; n=312), } \\
\% \text { (n) }\end{array}$ & $\begin{array}{l}\text { Группа КЦ } \\
\text { (8 мг/сут.; n=323), } \\
\% \text { (n) }\end{array}$ & $\mathrm{p}$ \\
\hline$<140 / 90$ мм рт. ст. & $55,8(174)$ & $48,8(157)$ & 0,077 \\
\hline$<135 / 80$ мм рт. ст. а & $26,9(84)$ & $19,6(63)$ & 0,028 \\
\hline$<130 / 85$ мм рт. ст. & $34,3(107)$ & $24,2(78)$ & 0,005 \\
\hline$<130 / 80$ мм рт. ст. & $25,3(79)$ & $18,3(59)$ & 0,033 \\
\hline$<125 / 80$ мм рт. ст. b & $19,9(62)$ & $14,3(46)$ & 0,061 \\
\hline
\end{tabular}

a) Целевые уровни АД по критериям JSH [12] b) Целевые уровни АД по критериям ESH/ESC [11] 

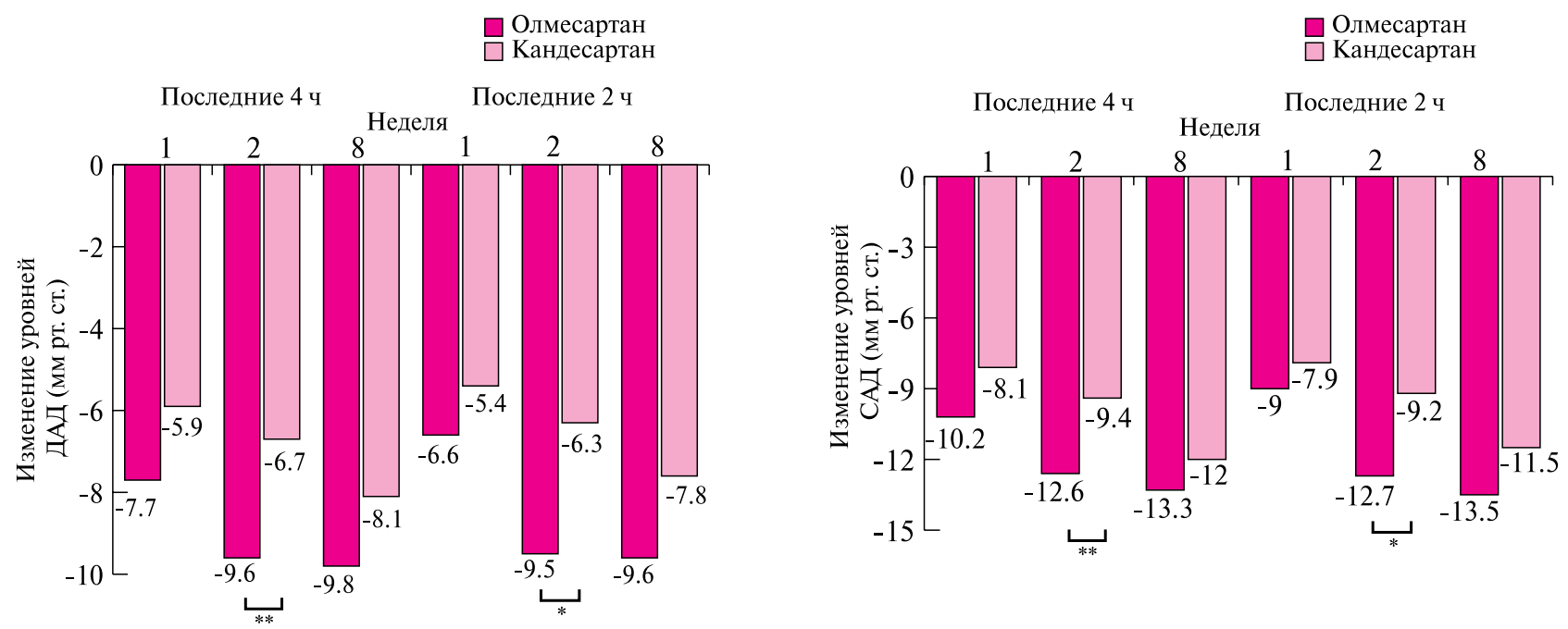

Примечание: (a) * $p=0,002 ; * * p=0,001 ;$ (b) * $p=0,007 ; * * p=0,004$.

Puc. 3 Среднее изменение уровней ДАД (a) и САД (b) в последние 4 и 2 ч СМАД на 1, 2 и 8 нед. терапии ОМ либо КЦ.

АД при лечении ОМ сохраняется и в ранние утренние часы - время, когда риск подъема АД особенно высок.

На основании полученных в конце 8 нед. лечения результатов СМАД оценивалось среднее снижение АД $_{24}$ и достижение целевых цифр АД по критериям ESH/ $\mathrm{ESC}$ и JSH [11,12]. Доля пациентов с достижением целевых уровней АД была достоверно выше в группе ОМ, чем в группе КЦ. Согласно критериям ESH/ESC, эти показатели составляли $25,6 \%$ vs $14,9 \%$, соответственно. Иными словами, терапия ОМ ассоциировалась с увеличением доли больных, достигнувших целевого АД, на 72 \%. К окончанию 8 недель лечения доля пациентов с достижением целевых цифр АДд по критериям ESH/ESC была вдвое выше в группе ОМ, чем в группе КЦ.

В норме АД изменяется в течение суток с достижением минимальных значений в ночное (н.) время. В ранние утренние часы ( с 03:00 до 06:00 [7]) АД начинает повышаться, и 06:00 наблюдается резкий подъем АД (morning surge) $[5,7]$. По результатам эпидемиологических исследований, частота ССС, таких как ИМ, МИ и внезапная сердеч-

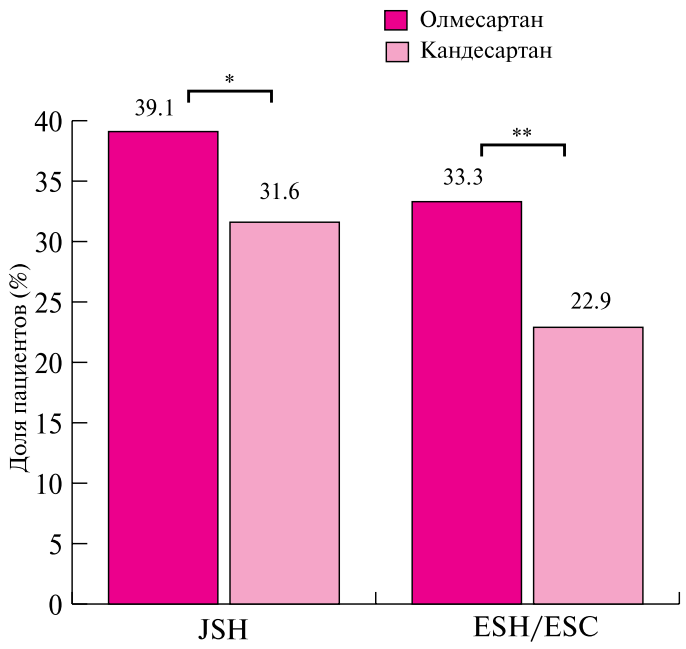

Примечание: $* \mathrm{p}=0,047 ; * * \mathrm{p}<0,001$.

Puc. 4 Доля пациентов с достижением целевых уровней АД в последние 4 ч СМАД на 8 нед. терапии ОМ или КЦ по критериям $\mathrm{ESH} / \mathrm{ESC}(<125 / 80$ мм рт.ст.; [11]) и JSH (<135/80 мм рт.ст.; [12]). ная смерть (ВСС), также достигает пика утром, в часы, следующие за УП АД [7,21,22]. Это позволяет предположить наличие причинно-следственной связи между УП АД и увеличением риска ССО. Как правило, АГП принимаются 1 раз в сутки, утром. Таким образом, УП АД совпадает c окончанием периода действия препарата. Оценка АД с помощью СМАД позволяет изучить эффективность АГТ на протяжении всех суток и, в т.ч. определить степень предупреждения УП АД. Согласно полученным результатам, ОМ (20 мг/сут.) обеспечивал более эффективное снижение АД в часы УП АД, чем КЦ (8 мг/сут.). Более выраженная антигипертензивная эффективность ОМ также подтверждалась большей долей больных с достижением целевых уровней АД в утренние часы, по критериям ESH/ESC и JSH. Доля пациентов, достигнувших целевых значений АД в последние 2 ч СМАД, была выше в группе ОМ, чем в группе КЦ.

В выполненном ранее, прямом, сравнительном исследовании официально рекомендуемых поддерживающих доз различных БРА было продемонстрировано, что целевые уровни давления, по данным СМАД, достигались у большей доли пациентов, принимавших ОМ, по сравнению с другими представителями этого класса препаратов [23]. В частности, доля участников, достигнувших целевых значений АД $24<130 / 80$ мм рт.ст., <130/85 мм рт.ст. и <140/90 мм рт.ст., была достоверно выше при приеме OM, чем при терапии лозартаном калия и валсартаном, а также выше, чем при лечении ирбесартаном [23]. Полученные результаты имеют важное клиническое значение не только потому, что ранее была подтверждена связь между снижением АД до целевых значений и уменьшением риска ССО [24,25], но и в связи с реальной возможностью достижения этих целевых уровней АД у большинства пациентов, как в условиях клинических исследований, так и в повседневной клинической практике [13,26-29].

Результаты анализа подтвердили более выраженную антигипертензивную эффективность ОМ в дозе 20 мг/сут., по сравнению с КЦ в дозе 8 мг/сут., в отношении стойкого снижения уровней АД 24 . Поскольку ОМ более эффективно предупреждал повышение УП АД, можно предположить, что это означает более выраженное снижение риска ССО, ассоциируемого с УП АД. 


\section{Заключение}

При лечении пациентов с АГ важно обеспечить эффективное снижение АД на протяжении суток, а также предупреждение УП АД, связанного с увеличением риска ССО Через 8 нед. терапии среднее снижение АД 24 в группе ОМ было более выраженным, чем в группе КЦ. Терапия ОМ ассоциировалась с более выраженным снижением ДАД и САД в последние 4 и 2 ч СМАД, а также с увеличением доли пациентов, у которых удалось добиться целевых значений давления в эти часы, согласно критериям ESH/ESC и JSH.

\section{Литература}

1. Ezzatl M, Lopez AD, Rodgers A, et al. Selected major risk factors andglobaland regional burdenof disease. Lancet 2002; 360: 1347-60.

2. Kannel WB. Blood pressure as a cardiovascular risk factor: preventionand treatment, JAMA 1996; 275: 1571-6.

3. World Health Organization. The WorldHealth Report, 2002.

4. White WB.Circadian variation of bloodpressure: clinical relevance and implicationsfor cardiovascularchronothcrapeutics. Blood Press Monit 1997; 2: 47-51.

5. Millar-CraigMW, CN Bishop, Raftery EB. Circadian variation of blood-pressure. Lancet 1978; I: 795-7.

6. Anwar YA, White WB. Chronotherapeutics for cardiovascular disease. Drugs 1998; 55: 631-43.

7. White WB. Cardiovascularrisk and therapeuticintervention for the early morningsurge inblood pressureand heart rate. Blood Press Monit 2001; 6: 63-72.

8. Neul B, MacMahon S, ChapmanN. Effects of ACE inhibitors, calciumantagonists, and other blood-pressure-lowering drugs: results of prospectively designed overviews of randomlsed trials. Blood Pressure Lowering Treatment Trialists' Collaboration. Lancet 2000; 356: 1955-64.

9. UKPDS. Tight bloodpressurecontroland risk of macrovascular and microvascularcomplications in type 2 diabetes: UKPDS 38. UK Prospective Diabetes Study Group. BMJ 1998; 317: 70313.

10, DahlofB, Sever PS, Poulter NR, et al. Prevention of cardiovascular events with an antihypertensive regimen of amlodipine adding perindopril as required versus atenolol adding bendroflurnethlazide as required, in the Anglo-Scandinavian Cardiac OutcomesTrinl-Blood PressureLoweringArm (ASCOT. BPLA) a multicentre randornised controlled trial. Lancet 2005; 366: 895-906.

11. European Society of Hypertension-European Society of Cardiology. 2003 European Society of Hypertension-European Society of Cardiology guidelines for the managementof arterial hypertension. J Hypertens 2003; 21: 1011-53.

12. Guidelines Committee. Japanese Society of Hypertension. 2004 Guidelines for the managementof hypertension (in Japanese). Life Science Publishing Co., Ltd 2004.

13. Chobanian AV, Bakris GL, Black HR, et al. The Seventh Report of the Joint National Committee on Prevention, Detection, Evaluation, and Treatment of High Blood Pressure: the JNC 7 report. JAMA 2003: 289: 2560-72.

14. McGrath BP. Is white-coat hypertension innocent? [Letter]. Lancet 1996; 348: 630 IS.

15. Clement DL, De Buyzerc ML, De Bacquer DA, et al. Prognostic value of ambulatory blood-pressure recordings inpatients with treated hypertension. N Engl J Med2003; 348: 2407-15.

16. Staessen JA. Thijs L, Fagard R, et al. Predicting cardiovascular risk using conventionalvs ambulatoryblood pressure in older patients with systolic hypertension, Systolic Hypertension in Europe Trial Investigators. JAMA J999; 282: 539-46.

\section{Благодарность}

Данное исследование было выполнено при поддержке компании Sankyo GmbH. Авторы также выражают благодарность г-ну Phil Jones (Adis Communications, по поручению Sankyo GmbH) за помощь в редактировании статьи. Д-р Brunner являлся членом экспертного комитета компании Sankyo GmbH, а также получал гонорары от Sankyo GmbH. Д-р Arakawa не имеет какого-либо конфликта интересов, связанного с выполнением данного исследования.

17. Maneia G, Parati G. Office compared with ambulatory blood pressure $1 n$ assesslug responseto antihypertensive treatment: a meta-analysis. J Hypertens 2004; 22: 435-45.

18. Brunner HR, Stumpe KO, Januszewicz A. Antihypertensive efficacy of olmesartan medoxomil and candesartan eilexetil assessedby 24-hour ambulatory blood pressure monitoring in patients with essential hypertension. Clin Drug Invest 2003; 23: 419-30.

19. Oparil S, Williams D, Chrysant SG, et al. Comparative efficacy of olmesartan, losartan, valsartan, and irbesartanin the control of essential hypertension. J Clin Hypertens 2001; 3: 283-91, 318.

20. Arakawa K. Significance of suppressing angiotensin by ARB [in Japanese, with abstract in English]. Progr Med 2004; 24: 175762 .

21. Muller JE, Stone PH, Turi ZG, et al. Circadian variation in the frequency of onset of acute myocardial infarction. $N$ Engl J Med 1985; 313: 1315-22.

22. Wllllch SN, Levy D, Rocco MD, et al. Circadian variation in the incidence of sudden cardiac death in the Framingham Heart Study population. Am J Cardio1 1987; 60: 801-6.

23. Smith DH, Dubiel R, Jones M. Useof 24-houl'ambulatory blood pressure monitoring to assess antihypertensive efficacy: a comparison of olmesartan medoxomil, losartanpotassium, valsartnn, and irbesnrtan. Am J Cardiovasc Drugs 2005; 5: 41-50.

24. Hansson L, Zanchetti A, Carruthers SG, et al. Effects of intensive blood-pressure lowering and low-dose aspirin in patients with hypertension: principal results of the Hypertension Optimal Treatment (HOT) randomlsed trial. HOT Study Group. Lancet 1998; 351: 1755-62.

25. Weber MA, Julius S, Kjeldsen SE, et al. Blood pressure dependent and independent effects of antihypertensive treatmenton clinical events in the VALUE trial. Lancet 2004; 363: 2049-51.

26. ALLHATO fficersand Coordinators for the ALLHAT Collaborative Research Group. The Antihypertensive and Lipid-Lowering Treatment to Prevent Heart Attack Trial. Major outcomes in high-risk hypertensive patients randomized to anglotensin-converting enzymeinhibitor or calcium channel blocker vs diuretic: The Antihypertensive lind Lipid-Lowering Treatment to Prevent Heart Attack Trial (ALLHAT). JAMA 2002; 288: 2981-97.

27. Dahlof B, Devereux RH, Kjeldsen SE, et al. Cardiovascular morbidity and mortality in the Losartan Intervention For Endpoint reduction in hypertension study (LIFE): a randomised trial against atenolol. Lancet 2002; 359: 995-1003.

28. Pepine CJ, Handberg EM, Cooper-DeHoff RM, et al. Acalcium antagonistvsa non-calcium antagonist hypertension treatment strategy for patients with coronary artery disease, The International Verapamil-Trandolapril Study (IN VEST): a randomized controlled trial. JAMA 2003; 290: 2805-16.

29. Singer GM, Izhar M, Black HR. Goal-oriented hypertension management: translating clinical trials to practice. Hypertension 2002; 40: 464-9. 